\title{
Disruption of temporal discrimination and the choose-short effect
}

\author{
RYAN D. WARD AND AMY L. OduM \\ Utah State University, Logan, Utah
}

\begin{abstract}
The present experiment examined the effects of several disruptors on temporal discrimination. Pigeons responded under a 0-delay symbolic matching-to-sample procedure in which responses to one key color were reinforced following the presentation of four shorter sample durations, and responses to another key color were reinforced following the presentation of four longer sample durations. Steady-state performance was disrupted by presession feeding, intertrial-interval food, visual distraction, and extinction. All disruptors decreased temporal-discrimination accuracy. Analyses of the fitted cumulative normal functions indicated that decreases in accuracy were produced mainly by decreases in overall stimulus control rather than specific effects on timing. In addition, all disruptors selectively decreased accuracy on long-sample trials - a choose-short effect. This effect is interpreted in terms of decreased attention to the samples under disruption. Current theories of the chooseshort effect do not appear to easily account for these results.
\end{abstract}

Discrimination processes are generally studied in nonhumans using some variant of the conditional-discrimination procedure (see Nevin, Davison, \& Shahan, 2005, for review). In this procedure, the response that will be reinforced depends on the occurrence of a prior sample event. A variety of manipulations disrupt steady-state discrimination performance. For example, delivering food during the intertrial interval decreases discrimination accuracy in delayed matching-to-sample (DMTS) procedures (see, e.g., Blough, 1998; Nevin, Milo, Odum, \& Shahan, 2003; Odum, Shahan, \& Nevin, 2005; Spetch, 1985) as does discontinuing reinforcers for correct choice responses (i.e., extinction; Nevin et al., 2003; Odum et al., 2005).

Blough (1996) proposed a theoretical framework with which such disruption might be characterized. He discussed three aspects of discrimination that affect performance: overall stimulus control, bias, and sensitivity. He suggested that in psychophysical procedures in which sample stimuli vary along some continuum, measures of these factors could be obtained by fitting a cumulative normal function with four parameters (upper and lower asymptotes, mean, and standard deviation) to the obtained psychophysical function. The difference between the upper and lower asymptotes of the function measures overall stimulus control and indicates the degree to which choice responses are under the functional control of the presented sample stimuli. The mean of the function measures bias and indicates whether choice responding is biased toward one response option over the other. The standard deviation $(S D)$ measures sensitivity and indexes confusion among stimuli or the degree of generalization that occurs between stimuli in the different categories.
Blough (1996) proposed that various experimental manipulations have separable effects on these three factors. He further suggested that such effects could be quantitatively differentiated by applying an asymptote correction that assumes perfect stimulus control (Heinemann, Avin, Sullivan, \& Chase, 1969) to the obtained psychophysical data before fitting the cumulative normal function. In several experiments, he demonstrated that various manipulations - such as increasing sample duration or introducing delays between samples and comparisons - indeed had separable effects on these three measures.

Using this theoretical framework, Blough (1998) characterized the disruptive effects of several different reinforcer manipulations on wavelength discrimination. Pigeons received food for pecking one response key following the presentation of six shorter wavelengths and for pecking another response key following the presentation of six longer wavelengths. Performance was characterized by plotting psychophysical functions relating the percentage of responses to the "long" wavelength response key to sample wavelength. During separate experimental conditions, reinforcers were presented during the intertrial interval (ITI), pecks to the sample key were reinforced, subjects were fed a large portion of their daily food intake prior to the session (prefeeding), and food for correct choice responses was discontinued for one session (extinction). Blough found significant decreases in stimulus control with ITI food and added reinforcers for sample key pecks. In addition, sensitivity decreased with added reinforcers for sample key pecks. Neither prefeeding nor extinction produced effects on stimulus control or sensitivity. When reinforcers for sample key pecks were added

R. D. Ward, ryanward@cc.usu.edu 
to extinction, however, both stimulus control and sensitivity decreased. These results suggest that the disruptive effects of various manipulations on stimulus control, bias, and sensitivity can be differentiated.

Such differentiation may be particularly useful when interpreting the effects of disruptive manipulations on behavior maintained by temporal-discrimination procedures. During a typical temporal-discrimination procedure, a sample is presented for some duration and the subject must categorize the presented sample as being either "short" or "long" by responding to some comparison option (e.g., lever or response key). A variety of manipulations disrupt performance under such procedures, including ITI food presentations, presession feeding (hereafter prefeeding), extinction, and visual distraction (Sutton \& Roberts, 2002; Ward \& Odum, 2006; Wilkie, Symons, \& Tees, 1988). The specific nature of such disruption, however, is unclear, since there have been no attempts to separate the effects of such manipulations on stimulus control, bias, and sensitivity of temporal discrimination using the analyses described by Blough (1996).

It is theoretically important that one separate the effects of various disruptive manipulations on the measures of stimulus control and bias, because current models of timing suggest that lateral shifts in the psychophysical function relating the proportion of "long" responses to sample duration (i.e., bias) result in part from changes in the speed of an internal pacemaker (see, e.g., Meck, 1996). McClure, Saulsgiver, and Wynne (2005), however, recently demonstrated that traditional methods of analyzing data from temporal-discrimination procedures - such as fitting a logistic function with two parameters (midpoint and slope) to the obtained proportion-long response data - can lead to inaccurate conclusions regarding changes in the estimates of bias and sensitivity. This outcome occurs because changes in the asymptotes of the function can affect the estimates of these parameters apart from any actual changes in the underlying function, particularly if the changes in the upper and lower asymptotes are asymmetric. For example, decreases in the upper asymptote of the function without corresponding increases in the lower asymptote can be reflected as changes in the bias parameter of a two-parameter model, even in the absence of lateral shifts in the function. Because of the theoretical import of changes in the measure of bias, fitting a model that yields unconfounded measures of stimulus control and bias under disruption conditions is desirable. The method suggested by Blough (1996) appears well suited to accomplish this goal.

An additional aim of the present experiment was to assess any differential effects of disruption on accuracy during short and long sample trials. When delays are inserted between samples and comparisons, accuracy on long sample trials declines sharply as a function of delay, whereas accuracy on short sample trials remains high across delays, a phenomenon known as the choose-short effect (see, e.g., Spetch \& Wilkie, 1982, 1983; see Spetch \& Rusak, 1992, for review). Using a psychophysical choice procedure, Fetterman (1995) assessed the differ- ential effects of delays on accuracy during short and long sample trials in which samples ranging from 2 to $10 \mathrm{sec}$ were presented. Delays between samples and comparisons $(0-15 \mathrm{sec})$ changed the asymptotes of the functions, indicating decreases in stimulus control. Particularly in Experiment 1, however, these changes were asymmetric. The proportion of long responses following long sample durations decreased as a function of delay, but the proportion of long choices following short sample durations did not increase - the typical choose-short effect.

A comparison of the data reported by Fetterman (1995) with the results of studies in which temporaldiscrimination performance has been disrupted by some other means indicates comparable effects of delays and other manipulations on the form of the psychophysical function. A variety of other manipulations result in similar asymptote shifts (see Ward \& Odum, 2006, for discussion). Such results, however, are generally not analyzed in terms of differential effects on short and long sample trials. Similar effects of delays and other disruptors on accuracy during short and long sample trials may indicate similar underlying mechanisms of disruption. Therefore, an additional aim of the present experiment was to assess the effects of several disruptors on accuracy during both short and long sample trials in a psychophysical choice procedure. Steady-state temporal-discrimination performance was disrupted by prefeeding, ITI food, distraction (see, e.g., Sutton \& Roberts, 2002), and extinction. The proportion-long response data obtained under disruption were then characterized in terms of effects on the measures of stimulus control, bias, and sensitivity (Blough, 1996) and on accuracy during both short and long sample trials.

\section{METHOD}

\section{Subjects}

Four White Carneau pigeons, with a history of exposure to a variety of unrelated experimental procedures, served as subjects. Pigeons were maintained at $80 \% \pm 15 \mathrm{~g}$ of their free-feeding weight by postsession feeding as needed. Between sessions, pigeons were individually housed with free access to water in a temperaturecontrolled colony under a 12:12 h light:dark cycle.

\section{Apparatus}

Four Lehigh Valley Electronics sound-attenuating chambers were used. Chambers were constructed of painted metal with aluminum front panels. The chambers measured $30 \mathrm{~cm}$ across, $35 \mathrm{~cm}$ deep, and $35 \mathrm{~cm}$ high. Each front panel had three translucent plastic keys that could be lit from behind with red, green, white, pink, and blue light and required a force of at least $0.10 \mathrm{~N}$ to record a response. Keys were $2.5 \mathrm{~cm}$ in diameter and $24 \mathrm{~cm}$ from the floor. A lamp (28 V, $1.1 \mathrm{~W}$ ) that was mounted $4.5 \mathrm{~cm}$ above the center key served as a houselight. A rectangular opening that was $8.5 \mathrm{~cm}$ below the center key provided access to a solenoid-operated hopper filled with pelleted pigeon chow. During hopper presentations, the opening was lit with white light and the houselight and keylights were extinguished. White noise and chamber ventilation fans masked extraneous noise. Contingencies were programmed and data were collected by a microcomputer using Med Associates interfacing and software.

\section{Procedure}

Because of the pigeons' previous experimental history, no keypeck training was necessary, and the experimental procedure began im- 
mediately. Sessions were conducted 7 days a week at approximately the same time. We used an interval bisection procedure during which the pigeons categorized the presented sample durations as being either of short or long duration by responding to a key that was lit in a corresponding color (see, e.g., Stubbs, 1968). Trials began with the illumination of the center key in red. This key served as a trial-ready stimulus. A response to the center key darkened it and lit the houselight for the duration of the presented sample. Following sample presentation, the left and right keys were lit blue and white, each key color corresponding to either a short or a long sample duration. For the purposes of the present experiment, durations shorter than $5 \mathrm{sec}$ were categorized as short, and durations longer than $5 \mathrm{sec}$ were categorized as long. The location of each color (left or right key) was randomly determined from trial to trial. A peck to the key that was lit in the color that corresponded to the duration of the temporal sample (short or long) resulted in 2.5-sec access to food. A peck to the key that was the other color produced a 2.5 -sec blackout. Key colors corresponding to short and long sample durations were counterbalanced across pigeons.

Training. The baseline procedure included eight sample durations $(2,3,4,4.5,5.5,6,7$, or $8 \mathrm{sec})$ that were selected randomly from trial to trial with the constraints that each sample duration appear once in each block of eight consecutive trials and that each sample duration be presented an equal number of times during the session. Initially, only sample durations of 2 and $8 \mathrm{sec}$ were presented. Once accuracy with these sample duration endpoints was at least $80 \%$ over the last five sessions, the next most extreme pair of sample durations was introduced. Once accuracy appeared stable over the last five sessions with these four sample durations, the next most extreme pair of sample durations was introduced. This training schedule continued until all eight of the sample durations were included in the daily sessions. If accuracy was low at any time during initial training as a result of a pronounced color or side bias, a correction procedure was implemented. During sessions in which the correction procedure was in place, a choice response to the key lit the color that did not correspond to the presented sample duration darkened both keys and initiated a 2.5 -sec blackout period, after which the entire trial was repeated with the same sample duration and comparison key colors in the same location. This process continued until a correct-choice response ended the trial in food. Three of the four pigeons required correction at some point during initial training, but correction was not required for the remainder of the experiment.

Baseline. Once accuracy with all eight sample durations was stable by visual inspection over the last five sessions, the probability of reinforcement for correct categorization of the six intermediate sample durations was decreased from 1.0 to 0.5 to allow more trials per session. The probability of reinforcement for correct categorization of the 2- and 8-sec sample endpoints remained 1.0. Trials were separated by a 20 -sec ITI during which all chamber stimuli were extinguished. Daily sessions ended after 64 trials; thus, each sample duration was presented eight times per session.

Disruption. After 20 sessions of baseline, performance was judged stable by visual inspection for all pigeons and disruption began. Disruption consisted of prefeeding, ITI food, distraction, and extinction (which will shortly be described in detail). All disruptors were in effect for five consecutive sessions. The order of exposure to each disruptor was counterbalanced across pigeons. Baseline was reestablished between each successive disruptor (20-25 sessions across pigeons).

Prefeeding. Pigeons were given $30 \mathrm{~g}$ of food in the homecage $1 \mathrm{~h}$ prior to their scheduled daily session.

ITI food. Random-time 20 -sec response-independent food was delivered during the ITI. Intertrial-interval food was scheduled by sampling a probability gate every $1 \mathrm{sec}$ during the ITI and delivering 2 -sec hopper presentations as appropriate to the sampled probability.

Distraction. The center key flashed on and off every $0.025 \mathrm{sec}$ during sample presentations. The color of the flashing center key was counterbalanced across pigeons. For pigeons 97 and 85, the color was green, and for pigeons 51 and 74 the color was pink.

Extinction. Reinforcers for correct choice responses were discontinued and all correct responses resulted in a 2.5 -sec blackout followed by the ITI. During the five extinction sessions, all pigeons completed all trials with the exception of P74 during the last three sessions. Data from all sessions for this pigeon were included, however, since at least half of the trials during the daily sessions were completed. Trials not completed were excluded from analyses.

\section{Data Analysis}

Baseline performance was characterized as the average of the last five sessions prior to the introduction of each disruptor. Temporaldiscrimination performance during baseline and under all disruption conditions was assessed by recording the proportion of responses to the key color that corresponded to the long sample duration following the presentation of all sample durations. These data were then plotted as a function of the presented sample duration and fit by a cumulative normal function with four parameters. The function fitted to the data was

$$
f(t)=a+\frac{b}{\sqrt{2 \pi \sigma}}\left[\exp -\left(\frac{(t-\mu)^{2}}{2 \sigma^{2}}\right)\right],
$$

where $f(t)$ is the proportion of long responses at a given stimulus duration $(t), a$ is the lower asymptote of the function, $b$ is the range of the function (upper - lower asymptote), $\mu$ the mean, and $\sigma$ the $S D$ (see, e.g., McClure et al., 2005). Fits were accomplished with the solver tool of the Microsoft Excel spreadsheet program.

Following the original analysis by Blough (1996), the parameters of the cumulative normal function are measures of three aspects of discrimination performance. The degree of overall stimulus control is reflected in the range of the function. A larger range indicates greater stimulus control, whereas decreases in the range indicate decreases in stimulus control. The mean is the point on the function where the proportion of responses to the long option is 0.5 . This measure is the point of subjective equality and quantifies lateral shifts in the function. Increases in the mean indicate shifts of the function to the right, whereas decreases indicate shifts to the left. Such shifts have been interpreted as reflecting under- or overestimation of time, respectively (see, e.g., Meck, 1996). The $S D$ (slope of the function) is a measure of sensitivity to time, with smaller $S D$ values indicating greater sensitivity to the differences between the short and long samples in the temporal-sample categories.

As was previously discussed, changes in the asymptotes of the function can influence estimates of the mean and $S D$. To obtain unconfounded estimates of these parameters, an asymptote correction (Heinemann et al., 1969) was applied to the obtained proportion long response data. The correction is given by

$$
p(R \mid A)=\frac{p(R)-p\left(R_{\mathrm{L}}\right)}{p\left(R_{\mathrm{U}}\right)-p\left(R_{\mathrm{L}}\right)},
$$

where $p(R \mid A)$ is the probability of a long response given perfect stimulus control, $p(R)$ is the obtained probability of a long response, $p\left(R_{\mathrm{U}}\right)$ is the value of the upper asymptote, and $p\left(R_{\mathrm{L}}\right)$ is the value of the lower asymptote. The assumption underlying this correction is that given perfect stimulus control, the obtained proportion long response values for the 2 - and 8 -sec sample durations should fall at 0 and 1.0, respectively. Failure to obtain these values indicates that stimulus control of choice responding by the temporal dimension of the sample stimuli was not perfect. This asymptote correction calculates a corrected proportion long response value for each obtained proportion long response value and gives estimates of the mean and $S D$ of the cumulative normal function with the assumption of perfect stimulus control. These estimates are therefore not confounded with changes in the upper and lower asymptotes of the function, and they are considered to represent more accurate measures of bias and sensitivity (see, e.g., Blough, 1996; Heinemann et al., 1969). All 
statistical comparisons were conducted with a criterion significance level of $p<.05$.

\section{RESULTS}

Figure 1 shows that the fits of the cumulative normal functions to the proportion long response data from successive conditions (averaged across pigeons) were uniformly excellent, with the function accounting for an average of $99.6 \%$ and $99.1 \%$ of the variance in the data from the baseline and disruption conditions, respectively. During all baseline conditions, the functions are sigmoid in shape and increase with increasing sample duration. These results indicate that choice behavior was under temporal control.

As shown in Figure 1, prefeeding (top panel) disrupted temporal discrimination, evidenced by a decrease in the
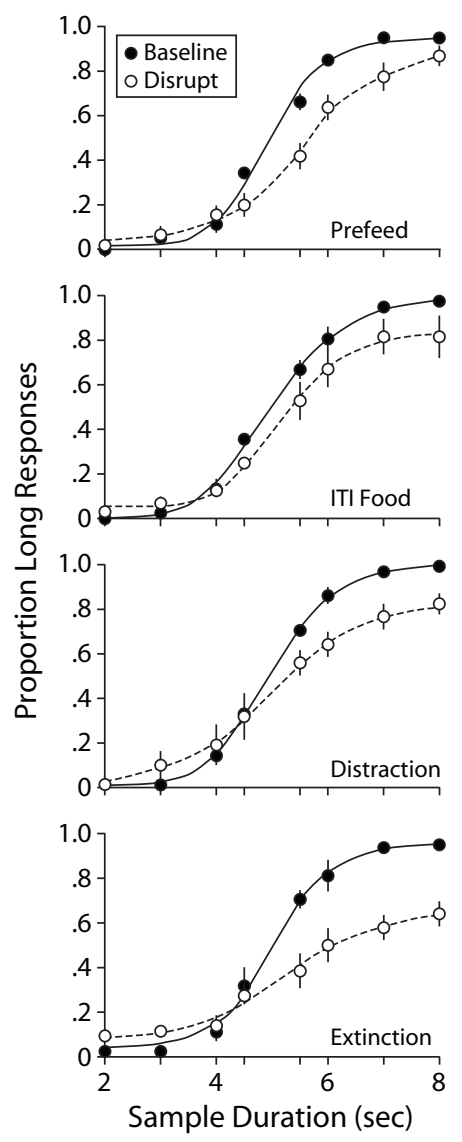

Figure 1. Mean proportion of responses to the key color corresponding to a long sample duration as a function of sample duration during all baseline and disruption conditions. In all panels, filled circles indicate baseline performance, whereas unfilled circles indicate performance during disruption. The solid and dashed lines in each panel are the best-fitting cumulative normal functions fitted to the data from the baseline and disruption conditions, respectively. In all cases, the proportion long response data from each respective baseline and disruption condition were averaged across pigeons, and one cumulative normal function was fit to the average data from each successive condition. Vertical bars represent one standard error above and below the mean. upper asymptote of the function and a general flattening of the function when compared with baseline. During ITI food, the function was somewhat flattened, with a particularly apparent decrease in the upper asymptote of the function. Distraction (third panel) and extinction (fourth panel) also flattened the functions and decreased the upper asymptotes of the function, with somewhat greater flattening occurring under extinction. There was also an apparent increase in the lower asymptote under extinction, whereas increases in the lower asymptote (if any) under other disruptors were relatively small.

Figure 2 shows fits of the cumulative normal function to the data from the baseline and disruption conditions for individual pigeons. The functions accounted for between $97.7 \%-99.9 \%$ and $79.6 \%-99.9 \%$ (across pigeons) of the variance from the baseline and disruption conditions, respectively. During the baselines preceding all disruption conditions, the functions for all pigeons were sigmoid and increased with increasing sample duration, indicating temporal control. All disruptors tended to flatten the functions, although the magnitude of this effect differed across pigeons. The upper asymptote of the functions decreased somewhat for all pigeons under all disruption conditions, although the magnitude of these decreases varied across pigeons and disruptors. The exception was for P51, under prefeeding. Increases in the lower asymptote also occurred in some cases, though these increases tended to be smaller than the corresponding decreases in the upper asymptote. In general, the average data in Figure 1 represent the individual performances well.

Figure 3 shows mean parameter estimates derived from fitting cumulative normal functions to the proportion long response data from individual pigeons across successive baseline and disruption conditions (shown in Figure 2). The top panel shows that under all baseline conditions, the ranges of the function were near $1.0(1.0-0.91$ across pigeons), indicating a high level of overall stimulus control. All disruptors decreased the range of the functions, indicating a decrease in stimulus control. Paired $t$ tests showed that the decrease in range was statistically significant following ITI food [ $t(3)=3.53]$, distraction $[t(3)=$ $3.46]$, and extinction $[t(3)=6.79]$, but not prefeeding $[t(3)=1.97]$.

The middle and bottom panels of Figure 3 show the parameter estimates of the measures of bias (the mean) and sensitivity (the $S D$ ), obtained after the asymptote correction (Equation 2) was applied to the obtained proportion long response data from individual pigeons (see Blough, 1996; Heinemann et al., 1969, for discussion). The estimates of the corrected mean (middle panel) were similar across successive baselines (4.7-5.1 sec across pigeons). The mean of the functions increased somewhat during three of four disruptors and decreased somewhat under distraction. Variability across subjects also increased under disruption. The changes in the mean under disruption were assessed by paired $t$ tests, which found no significant effects of disruption during prefeeding $[t(3)=$ 2.43], ITI food [ $t(3)=2.15]$, distraction $[t(3)=0.33]$, or extinction $[t(3)=1.06]$. 

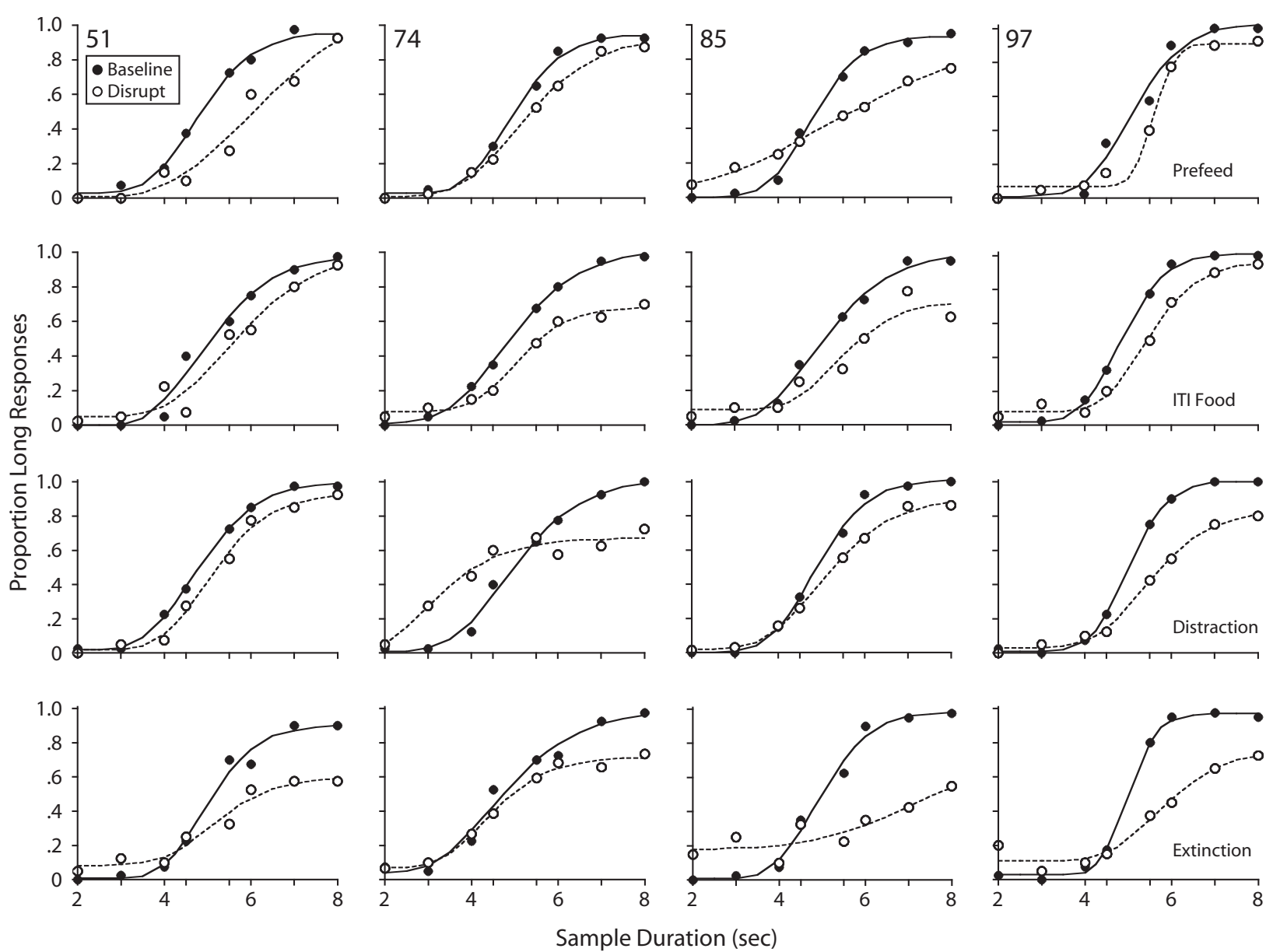

Figure 2. Fits of the cumulative normal functions to the proportion long response data from individual pigeons. In all cases, one function was fit to the average proportion long response data from each successive baseline and disruption condition for each pigeon. Filled circles indicate baseline performance, whereas unfilled circles indicate performance during disruption. The solid and dashed lines in each panel are the best-fitting cumulative normal functions fitted to the data from the baseline and disruption conditions, respectively.

As with the other parameters, the estimates of the corrected $S D$ (bottom panel) under all successive baselines were similar (1.1-1.3 across pigeons). The $S D$ increased somewhat under three of four disruptors, as did variability across subjects. Paired $t$ tests, however, indicated that these changes were not significant following prefeeding $[t(3)=1.15]$, ITI food $[t(3)=1.07]$, distraction $[t(3)=$ $2.03]$, or extinction $[t(3)=1.16]$.

\section{Time Course of Disruption}

Figures 1 and 2 show that all disruptors decreased temporal-discrimination accuracy, and Figure 3 shows that this disruption was largely produced by selective decreases in stimulus control. Examining the time course across successive sessions of disruption on accuracy during short and long sample trials may have utility for understanding the processes mediating the decreases of stimulus control. Figure 4 shows accuracy (calculated as percent correct) on both short and long sample trials across the five sessions of baseline preceding disruption and across the five sessions of each disruptor. During the baselines, accuracy on both short- and long-sample trials was generally high and stable. Under all disruption conditions, accuracy on short-sample trials remained high, whereas accuracy on long-sample trials decreased. Twofactor (sample duration $\times$ session) repeated measures ANOVAs found a significant effect of duration (short vs. long) during prefeeding $[F(1,3)=33.30]$, ITI food $[F(1,3)=18.40]$, and extinction $[F(1,3)=19.66]$, but not during distraction $[F(1,3)=3.02]$. The lack of a significant effect of duration under distraction likely reflects the fact that long-sample trial accuracy increased across the five sessions of disruption to the point where it was indistinguishable from accuracy on short-sample trials by Session 4 (see below).

Although all disruptors tended to decrease accuracy on long-sample trials, the character of disruption differed between disruptors. For both prefeeding and ITI food, disruption decreased accuracy during the first session, after which accuracy remained relatively stable. By contrast, during distraction, accuracy decreased substantially during the first session, but increased across the remainder 

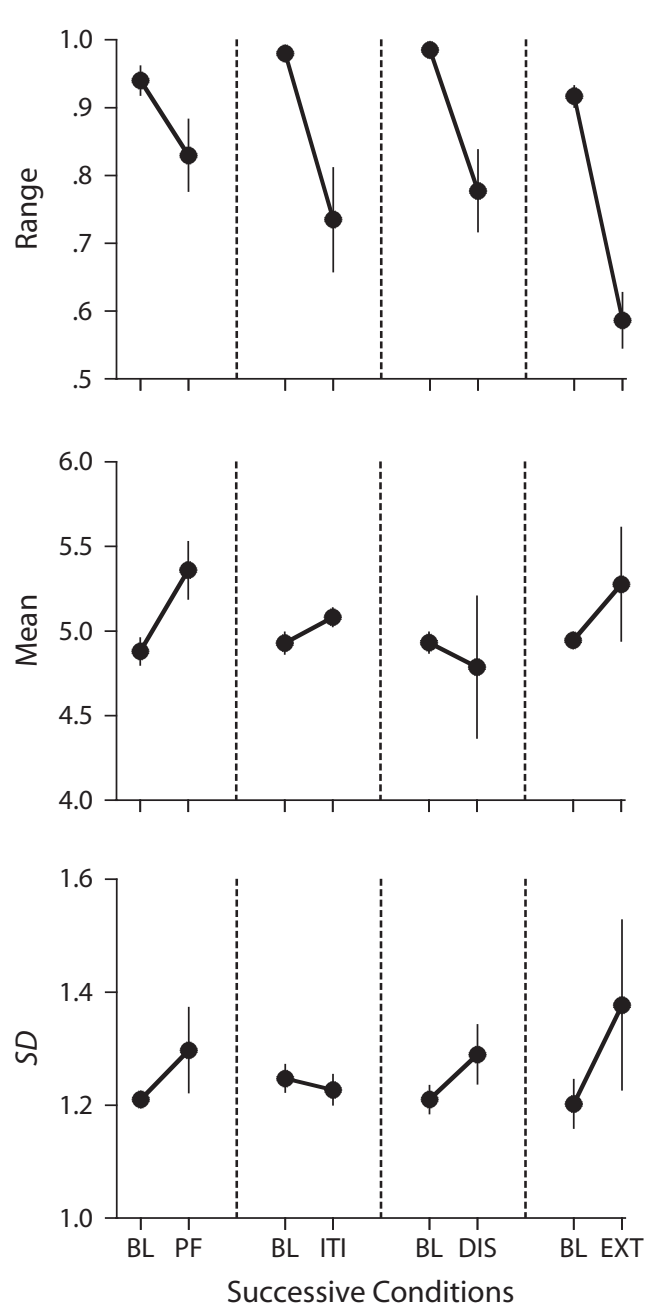

Figure 3. Mean parameter estimates from the fit of the cumulative normal function (see text for details) to mean proportion long responses across successive baseline and disruption conditions. In all cases, the function was fit to the average data from each successive baseline and disruption condition for each pigeon, and the resulting parameter estimates were averaged. The top panel shows the range (upper asymptote - lower asymptote) of the function. The middle panel shows corrected estimates of the mean. The bottom panel shows corrected estimates of the $S D$ of the function. Vertical bars represent one standard error above and below the mean. Abbreviations along the $x$-axis indicate baseline (BL), prefeeding (PF), intertrial-interval food (ITI), distraction (DIS), and extinction (EXT).

of the disruption sessions. Under extinction, accuracy declined sharply across the five sessions of disruption. The ANOVAs failed to find a significant effect of session during prefeeding $[F(4,12)=0.85]$ or ITI food $[F(4,12)=$ 1.21]. Significant effects of session were, however, obtained during distraction $[F(4,12)=4.66]$ and extinction $[F(4,12)=13.76]$. The interaction between duration and session was not significant under prefeeding or ITI food, but approached conventional levels of significance under distraction $(p=.07)$ and extinction $(p=.06)$.

Figure 4 shows that decreases in accuracy across disruption sessions were due almost entirely to performance decrements on long-sample trials. In addition, inspection of the psychophysical functions (Figures 1 and 2) shows that sample durations near the category boundary of $5 \mathrm{sec}$ (e.g., 4.5 and $5.5 \mathrm{sec}$ ) were inaccurately categorized more often than sample durations near 2 and $8 \mathrm{sec}$, indicating greater discriminability of short and long samples nearer these endpoints. This difference in discriminability might be reflected in more incorrect choice responses on longsample trials in which sample stimuli nearer the short-long category boundary were presented. To assess this possibility, we calculated the percent correct for the short and long samples in each sample-duration pair (2-8 sec, $3-7 \mathrm{sec}$, 4-6 sec, 4.5-5.5 sec) during each respective baseline and disruption condition. Figure 5 shows the results of this analysis. During all baselines, accuracy was higher on trials in which one of the sample endpoints $(2$ or $8 \mathrm{sec})$ was presented and decreased as sample durations approached the category boundary. In addition, there was no systematic difference between accuracy on short- or long-sample trials. Under disruption, accuracy decreased as sample durations approached the boundary, but accuracy on shortsample trials was higher than accuracy on long-sample trials at all sample-duration pairs. In addition, the difference between accuracy on short- and long-sample trials appeared to be the most pronounced near the category boundary. Two-factor (sample pair $\times$ duration) repeated measures ANOVAs found significant effects of sample pair under all disruptors: prefeeding $[F(3,9)=56.41]$, ITI food $[F(3,9)=14.77]$, distraction $[F(3,9)=17.23]$, and extinction $[F(3,9)=26.77]$. Significant effects of sample duration (short vs. long) were also noted for prefeeding $[F(1,3)=39.13]$, ITI food $[F(1,3)=27.72]$, and extinction $[F(1,3)=24.64]$, but not for distraction $[F(1,3)=$ 3.88]. In addition, the interaction between pair and duration was significant under prefeeding $[F(3,9)=4.46]$ and ITI food $[F(3,9)=6.64]$, but not under distraction $[F(3,9)=0.05]$ or extinction $[F(3,9)=0.70]$. These results suggest that all disruptors decreased accuracy on long-sample trials more so than accuracy on short-sample trials at all sample pairs (although the decrease was not significant during distraction). Furthermore, in some cases, as the difficulty of the discrimination increased, the tendency to respond to the option corresponding to the short-sample duration also increased.

To further examine the effects of the disruptors on temporal-discrimination performance, we calculated choice-response latencies. Increases in choice-response latencies could function as a variable delay between samples and comparisons, possibly producing selective disruption of accuracy on long-sample trials. Figure 6 shows that during all baselines, the median latency to respond to a choice comparison was around $1 \mathrm{sec}(0.54-1.35 \mathrm{sec}$, across pigeons). Choice-response latency was not systematically affected under disruption. Because choice-response latencies were not normally distributed, we evaluated the changes under disruption using nonparametric statistics. Wilcoxon signedranks tests found no significant changes in the choiceresponse latencies under prefeeding $(W=-10)$, ITI food $(W=0)$, distraction $(W=5)$, or extinction $(W=-8)$. 

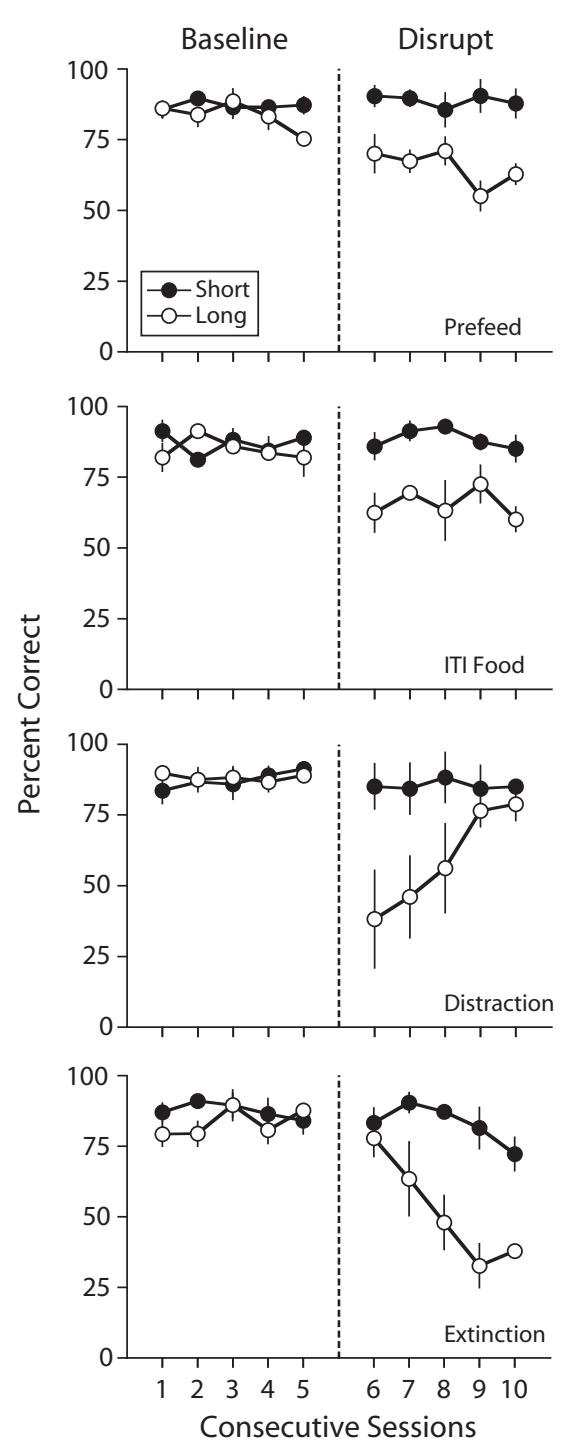

Figure 4. Mean percent correct (averaged across pigeons) on short $(2,3,4$, and $4.5 \mathrm{sec})$ and long $(5.5,6,7$, and $8 \mathrm{sec})$ sample trials across the last five sessions of each baseline and across all five sessions of each disruption condition.

\section{DISCUSSION}

During all baselines, behavior showed control by the temporal samples. All disruptors flattened the psychophysical functions, indicating a general disruption of temporal discrimination. These results replicate those of previous studies (see, e.g., Killeen, Hall, \& Bizo, 1999; Sutton \& Roberts, 2002; Ward \& Odum, 2006). In addition, analysis of the cumulative normal functions indicated all manipulations disrupted temporal discrimination by selectively decreasing overall stimulus control. The corrected measures of bias and sensitivity - the mean and $S D$ of the fitted functions - were not significantly affected. These results suggest that although choice responses under disruption were not always under functional control of the presented sample durations, sensitivity to the short- and long-sample durations remained high and disruption did not produce robust overestimation or underestimation of the duration of the sample stimuli.

The aim of the present analysis was to separate the effects of the disruptors on stimulus control from those on bias. The four-parameter model was chosen for this reason. A two-parameter model with only terms for bias and sensitivity also fit the data well, but does not allow for the crucial separation of effects on stimulus control and bias. With a two-parameter model analysis, the present effects are reflected largely as changes in bias. Such an interpretation is tenable when overall stimulus control is complete, but the data presented in Figures 1 and 2 clearly show that overall stimulus control was incomplete, particularly under disruption. Thus, although the four-parameter model is more complex than the two-parameter model, the added complexity may lead to more accurate characterization of the present results.

The results of the ITI food disruption replicate and extend those of Blough (1998). Blough reported that ITI food decreased overall stimulus control by wavelength, and the present results replicate this effect using temporal-sample stimuli. Two results from the present study, however, differ from those reported by Blough. He reported that neither prefeeding nor extinction had any effects on overall stimulus control. In the present study, however, both prefeeding and extinction decreased stimulus control (although these decreases were not significant during prefeeding).

The difference between the results of extinction in the present study and those reported by Blough (1998) may be due to procedural differences between experiments. Under the baseline preceding extinction in Blough's procedure, reinforcement was scheduled for $5 \%$ of correct-choice responses (36 out of 720 trials), whereas in the present experiment, reinforcement was available for about $63 \%$ of correct-choice responses ( 40 out of 64 trials). The difference in trial numbers and reinforcement probability between experiments means that the reduction in reinforcement produced by extinction in the present procedure likely was much more evident than that in the experiment reported by Blough, possibly contributing to the greater disruption observed in the present procedure. As for the differences in the effects of prefeeding, in the present study, we found only modest disruption (compared with the effects of the other disruptors) after prefeeding $30 \mathrm{~g}$ per day, whereas Blough reported no effects after prefeeding $12 \mathrm{~g}$ per day. Perhaps the amounts that were prefed contributed to the somewhat different results across studies.

Analyses of the psychophysical functions indicated that all disruptors produced performance decrements mainly by decreasing stimulus control rather than by affecting timing (bias). Similar results have been recently reported following administration of both D-amphetamine (McClure et al., 2005) and morphine (Ward \& Odum, 2005). These results, together with the many discrepant results in the literature on the effects of drugs on timing (see McClure et al., 2005; Odum, Lieving, \& Schaal, 2002, for review and discussion), suggest that drugs may produce their effects through a general disruption of stimulus con- 


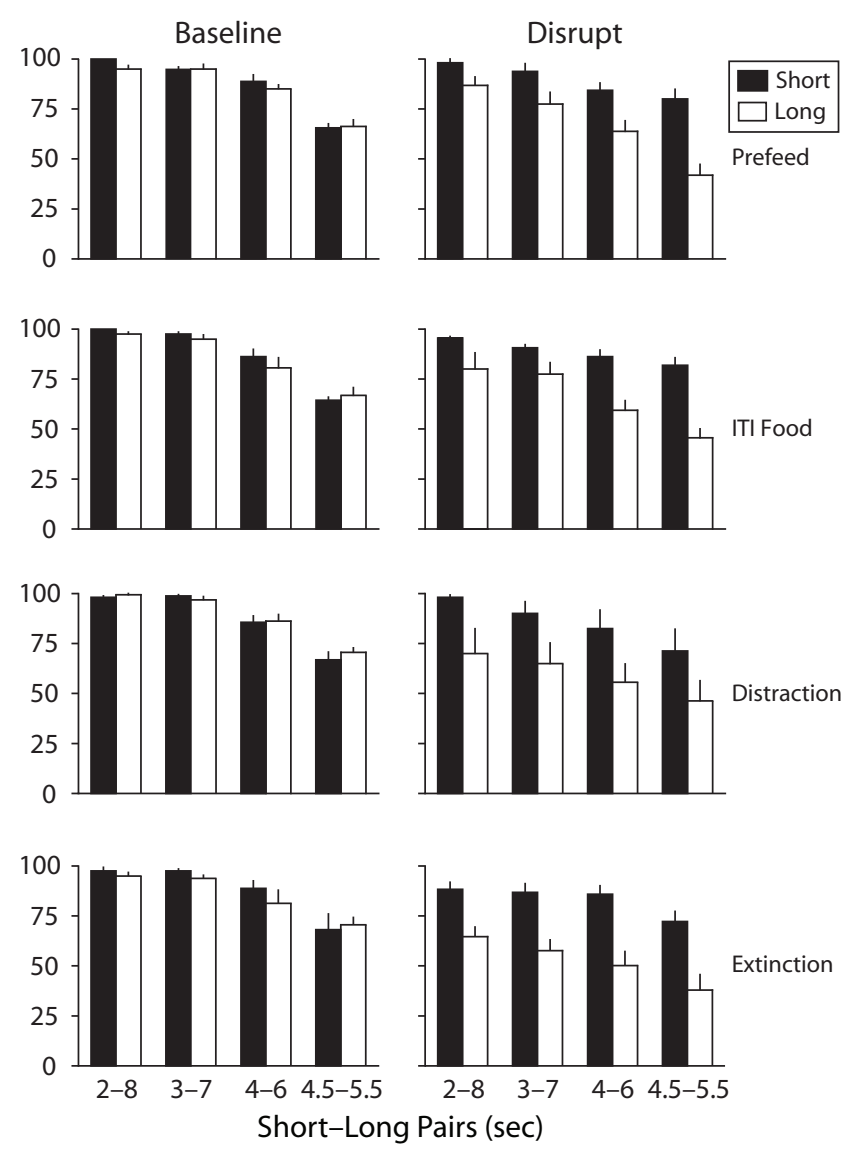

Figure 5. Mean percent correct (averaged across pigeons) during short and long sample trials for all sample-duration difficulty pairs during all baseline and disruption conditions. Vertical bars represent one standard error above the mean.

trol that is unrelated to any specific effects on timing, and these effects may result when steady-state temporal discrimination performance is disrupted by any number of manipulations (see, e.g., Howell, Byrd, \& Marr, 1986; see Ward \& Odum, 2005, 2006, for discussion).

The asymmetric changes in the asymptotes of the functions under all disruptors, as well as the data in Figures 4 and 5, indicate that all disruptors selectively decreased accuracy on long-sample trials - a choose-short effect. The most prominent theory of this effect, known as the subjective-shortening hypothesis (Spetch \& Wilkie, 1983), suggests that the remembered duration of the sample decreases as delays between sample presentation and presentation of the comparisons increase. When the comparison stimuli are presented, the remembered duration is shorter than the actual presented duration, and choice responses to the short option are more likely (see, e.g., Spetch \& Rusak, 1992; Spetch \& Wilkie, 1983). The present results, however, do not appear explicable by this account, because there were no programmed delays between samples and comparisons (and choice-response latencies were not systematically affected under disruption) in the present study.
Another, more recent account of the choose-short effect is the ambiguity hypothesis (see, e.g., Sherburne, Zentall, \& Kaiser, 1998; Zentall, 2006). In many DMTS procedures, the delay and ITI are not differentiated (they are both dark). The ambiguity hypothesis suggests that the choose-short effect occurs under these conditions because of confusion between the delay and ITI. When the similarity of the delay and ITI is decreased by lighting one or the other, the choose-short effect is often eliminated (see, e.g., Sherburne et al., 1998; Spetch \& Rusak, 1992). The present findings could not result from difficulty in differentiating between delays and the ITI, however, because no delays were present. Thus, it appears that neither the subjective shortening nor the ambiguity hypothesis can easily explain the present results. It is possible, however, that different manipulations (retention intervals vs. other disruptors) produce the choose-short effect in different ways.

One way in which the present manipulations might produce the choose-short effect is through a disruption of attention to the samples. The analyses of the psychophysical functions indicated that all disruptors selectively decreased stimulus control. Attention to samples is an important as- 


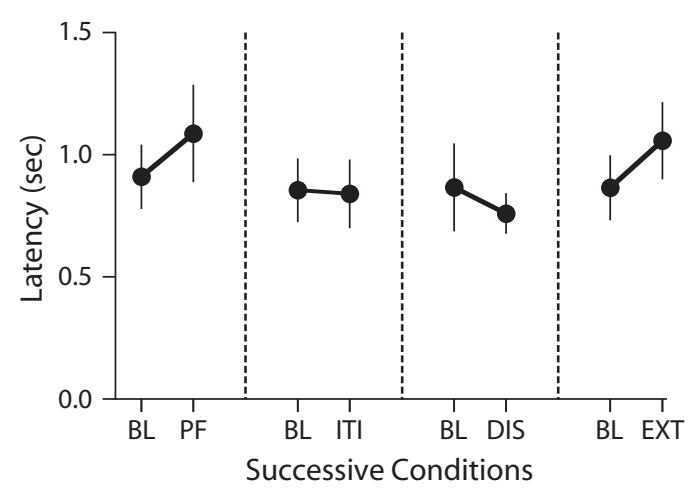

Figure 6. Median choice-response latency (averaged across pigeons) during successive baseline and disruption conditions. Other details as in Figure 3.

pect of the development and maintenance of stimulus control in discrimination procedures (see, e.g., Heinemann \& Avin, 1973; McIlvane, Dube, \& Callahan, 1996). In fact, in their original and subsequent discussions, Heinemann and colleagues (e.g., Heinemann et al., 1969) referred to the asymptote correction (Equation 2) as the correction for inattention. They assumed that performance decrements at the endpoints of the sample-stimulus continuum ( 2 and $8 \mathrm{sec}$ in this case) during steady-state performance result from inattention to the sample stimuli. Following this logic, if performance decrements under steady state were assumed to reflect some constant level of inattention, such decrements would be presumably magnified if some manipulation directly disrupted attention.

In other discrimination procedures (e.g., color matching), disrupting attention to samples would not be expected to increase choices of one comparison stimulus over the other (e.g., red or green). In temporal-discrimination procedures, however, such disruption could be predicted to produce more "short" choices. For example, if an 8-sec sample is presented and not attended to for $5 \mathrm{sec}$, the remaining $3 \mathrm{sec}$ of the sample - if attended to - will likely be categorized as short. Furthermore, if the first few seconds of a sample are not attended to, or if attending to the sample is intermittent, the tendency to respond "short" should increase following the presentation of longduration samples that are closer to the short-long category boundary $(5 \mathrm{sec})$. This effect would occur because as longduration samples get shorter, the attended-to portion of the sample will more likely fall into the "short" category. The data presented in Figure 5 provide some support for this assumption. This interpretation is in accord with the dominant account of timing - scalar expectancy theory (SET; Gibbon, 1977). According to SET, at the beginning of a sample presentation a switch is closed, which allows pulses to be gated from a pacemaker into an accumulator. Disruption of attention is suggested to increase the latency to close the switch, resulting in fewer pulses in the accumulator and sample durations being consistently categorized as shorter than the nominal duration (see, e.g., Lejuene, Macar, \& Zakay, 1999, for discussion).
An increase in responses to the short option also might be predicted if samples were not attended to at all under disruption. Several studies have shown that when no sample is presented, choice responses tend to favor the response option correlated with the less salient (e.g., sample stimulus of shorter duration, sample stimulus with fewer key pecks) of the two sample stimuli (see, e.g., Church, 1980; Fetterman \& MacEwen, 1989; Santi \& Hope, 2001; Spetch \& Wilkie, 1983). Wixted and his colleagues (Dougherty \& Wixted, 1996; Gaitan \& Wixted, 2000; Wixted \& Gaitan, 2004) offered a detection hypothesis to account for such effects. According to this account, when sample stimuli differ in salience (e.g., short vs. long, few vs. many, no food vs. food), subjects could make choice responses on the basis of the presence or absence of the more salient sample stimulus (see Grant, 1991; Sherburne \& Zentall, 1993, for related discussion). Thus, if samples were not attended to at all on some trials under disruption in the present study, the perceived absence of a long sample may have prompted the pigeons to respond to the short option.

Considerable debate has centered on the nature and role of the memory code for temporal samples in producing the choose-short effect (see Grant, Spetch, \& Kelly, 1997, for review). Some suggest that the memory code is analogi$\mathrm{cal}$, and pigeons retrospectively evaluate the duration of the sample at the choice point (see, e.g., Spetch \& Wilkie, 1983; Talarico \& Grant, 2006; Wilkie \& Willson, 1990). Others suggest that sample duration is coded categorically (i.e., "short" or "long") and that subjects base choice responses on prospective cues (i.e., "if short peck white, if long peck blue"; Kraemer, Mazmanian, \& Roberts, 1985). The interpretation of the choose-short effect in the present study differs from these accounts in that disrupting attention to the samples likely produces its effects before sample durations are coded, whereas memory-based theories suggest that the choose-short effect is produced by changes in the already-coded memory for the sample. The present results, therefore, appear consistent with both analogicalretrospective and categorical-prospective coding accounts, and future research is needed to determine the specific nature and role of the memory code in the manifestation of the choose-short effect in the present procedure.

The present demonstration of a choose-short effect in a 0 -delay temporal-discrimination procedure may pose difficulties for contemporary theories of the choose-short effect. Neither the subjective-shortening account nor the ambiguity hypothesis seem adequate as currently formulated, because there were no delays between samples and comparisons in the present procedure. Other accounts of the choose-short effect that posit diminishing trace strength (e.g., tuned-trace multiple-time-scale model; Staddon \& Higa, 1999) or changes in conditioned behavioral states (behavioral theory of timing; Killeen \& Fetterman, 1988) during retention intervals appear similarly unable to explain the present data. While the detection hypothesis appears to be consistent with the results of the present experiment, it has only formally been applied to the results from studies in which accuracy is assessed at 
different retention intervals, and conclusions about its applicability to the present data may therefore be premature. Thus, the detection hypothesis as well as other current theories of the choose-short effect may require revision to account for the results of the present experiment.

Alternatively, as was noted previously, the choose-short effect in the present study may be the result of different processes than the choose-short effect traditionally obtained in DMTS studies. Indeed, inspection of the data in Figure 4 suggests that the mechanism of this effect within the present study possibly differed between disruptors. The effects of prefeeding, ITI food, and extinction possibly reflect a decrease in motivation to attend to the samples, whereas the effect of distraction may have been to divert attention away from the samples (and this effect diminished across sessions). Whatever the nature of these differences, and whether the present disruption was produced by decreasing attention to the samples or by some other mechanism, the present results suggest that under some conditions the choose-short effect could result from a general disruption of temporal discrimination that is not mediated by changes in memory for sample duration (see Dorrance, Kaiser, \& Zentall, 2000; Zentall, 2006).

\section{AUTHOR NOTE}

The authors thank Chris Podlesnik, Corina Jimenez-Gomez, and Tim Shahan for comments on a previous version of this article and also Tiffany Rees and Cheryl Newbold for help in conducting the research. Address correspondence to R. D. Ward or A. L. Odum at the Department of Psychology, 2810 Old Main Hill, Utah State University, Logan, UT, 84322-2810 (e-mail: ryanward@cc.usu.edu or amy.odum@usu.edu).

\section{REFERENCES}

Blough, D. S. (1996). Error factors in pigeon discrimination and delayed matching. Journal of Experimental Psychology: Animal Behavior Processes, 22, 118-131.

BLOUGH, D. S. (1998). Context reinforcement degrades discriminative control: A memory approach. Journal of Experimental Psychology: Animal Behavior Processes, 24, 185-199.

CHURCH, R. M. (1980). Short-term memory for time intervals. Learning \& Motivation, 11, 208-219.

Dorrance, B. R., Kaiser, D. H., \& Zentall, T. R. (2000). Event duration discrimination by pigeons: The choose-short effect may result from retention-test novelty. Animal Learning \& Behavior, 28, 344-353.

Dougherty, D. H., \& Wixted, J. T. (1996). Detecting a nonevent: Delayed presence-versus-absence discrimination in pigeons. Journal of the Experimental Analysis of Behavior, 65, 81-92.

FETTERMAN, J. G. (1995). The psychophysics of remembered duration. Animal Learning \& Behavior, 23, 49-62.

FetTERMan, J. G., \& MACEwEN, D. (1989). Short-term memory for responses: The "choose-small" effect. Journal of the Experimental Analysis of Behavior, 52, 311-324.

GAITAN, S. C., \& WIXTED, J. T. (2000). The role of "nothing" in memory for event duration in pigeons. Animal Learning \& Behavior, 28, $147-161$.

Gibbon, J. (1977). Scalar expectancy theory and Weber's law in animal timing. Psychological Review, 84, 279-325.

GRANT, D. S. (1991). Symmetrical and asymmetrical coding of food and no-food samples in delayed matching in pigeons. Journal of Experimental Psychology: Animal Behavior Processes, 17, 186-193.

Grant, D. S., Spetch, M. L., \& Kelly, R. (1997). Pigeons' coding of event duration in delayed matching-to-sample. In C. M. Bradshaw \&
E. Szabadi (Eds.), Time and behavior: Psychological and neurobehavioural analyses (pp. 217-264). Amsterdam: Elsevier.

Heinemann, E. G., \& Avin, E. (1973). On the development of stimulus control. Journal of the Experimental Analysis of Behavior, 20, 183-195

HeinemanN, E. G., Avin, E., Sullivan, M. A., \& Chase, S. (1969). Analysis of stimulus generalization with a psychophysical method. Journal of Experimental Psychology, 80, 215-224.

Howell, L. L., BYrd, L. D., \& MARR, M. J. (1986). Similarities in the rate-altering effects of white noise and cocaine. Journal of the Experimental Analysis of Behavior, 46, 381-394.

KilleEn, P. R., \& FetTerman, J. G. (1988). A behavioral theory of timing. Psychological Review, 95, 274-295.

KilleEN, P. R., HAll, S., \& BizO, L. A. (1999). A clock not wound runs down. Behavioural Processes, 45, 129-139.

Kraemer, P. J., Mazmanian, D. S., \& Roberts, W. A. (1985). The choose-short effect in pigeon memory for stimulus duration: Subjective shortening versus coding models. Animal Learning \& Behavior, 13, 349-354.

LeJUene, H., Macar, F., \& ZaKay, D. (1999). Attention and timing: Dual-task performance in pigeons. Behavioural Processes, 45, 141-157.

McClure, E. A., Saulsgiver, K. A., \& Wynne, C. D. L. (2005). Effects of D-amphetamine on temporal discrimination in pigeons. $B e-$ havioural Pharmacology, 16, 193-208.

McIlvane, W. J., Dube, W. V., \& Callahan, T. D. (1996). Attention: A behavior analytic perspective. In G. R. Lyon \& N. A. Krasnegor (Eds.), Attention, Memory, and Executive Function (pp. 97-117). Baltimore: Paul H. Brookes.

Меск, W. H. (1996). Neuropharmacology of timing and time perception. Brain Research: Cognitive Brain Research, 3, 227-242.

Nevin, J. A., Davison, M., \& Shahan, T. A. (2005). A theory of attending and reinforcement in conditional discrimination. Journal of the Experimental Analysis of Behavior, 84, 281-303.

Nevin, J. A., Milo, J., Odum, A. L., \& Shahan, T. A. (2003). Accuracy of discrimination, rate of responding, and resistance to change. Journal of the Experimental Analysis of Behavior, 79, 307-321.

ODUM, A. L., LiEVING, L. M., \& SchaAL, D. W. (2002). Effects of $\mathrm{D}$-amphetamine in a temporal discrimination procedure: Selective changes in timing or rate dependency? Journal of the Experimental Analysis of Behavior, 78, 195-214.

OdUM, A. L., Shahan, T. A., \& Nevin, J. A. (2005). Resistance to change of forgetting functions and response rates. Journal of the Experimental Analysis of Behavior, 84, 65-75.

SANTI, A., \& HOPE, C. (2001). Errors in pigeons' memory for number of events. Animal Learning \& Behavior, 29, 208-220.

SherburNe, L. M., \& Zentall, T. R. (1993). Coding of feature and no-feature events by pigeons in a delayed conditional discrimination. Animal Learning \& Behavior, 21, 92-100.

Sherburne, L. M., Zentall, T. R., \& KAISER, D. H. (1998). Timing in pigeons: The choose-short effect may result from "confusion" between delay and intertrial intervals. Psychonomic Bulletin \& Review, 5, 516-522.

SPETCH, M. L. (1985). The effect of intertrial interval food presentations on pigeons' delayed matching to sample accuracy. Behavioural Processes, 11, 309-315.

SPETCH, M. L., \& RUSAK, B. (1992). Time present and time past. In W. K. Honig \& J. G. Fetterman (Eds.), Cognitive aspects of stimulus control (pp. 47-67). Hillsdale, NJ: Erlbaum.

SPeTCH, M. L., \& WiLKIE, D. M. (1982). A systematic bias in pigeons' memory for food and light durations. Behaviour Analysis Letters, 2, 267-274.

Spetch, M. L., \& WiLKIE, D. M. (1983). Subjective shortening: A model of pigeons' memory for event duration. Journal of Experimental Psychology: Animal Behavior Processes, 9, 14-30.

STADDON, J. E. R., \& HIGA, J. J. (1999). Time and memory: Towards a pacemaker-free theory of interval timing. Journal of the Experimental Analysis of Behavior, 71, 215-251.

STUBBS, D. A. (1968). The discrimination of stimulus duration by pigeons. Journal of the Experimental Analysis of Behavior, 11, 223-238. 
Sutton, J. E., \& Roberts, W. A. (2002). The effect of nontemporal information processing on time estimation in pigeons. Learning \& Motivation, 33, 123-140.

TAlarico, D. C., \& Grant, D. S. (2006). Effect of training delays and start and stop markers on the choose-short effect in pigeons. Behavioural Processes, 71, 98-106.

WARD, R. D., \& ODUM, A. L. (2005). Effects of morphine on temporal discrimination and color matching: General disruption of stimulus control or selective effects on timing? Journal of the Experimental Analysis of Behavior, 84, 401-415.

WARD, R. D., \& ODUM, A. L.(2006). Effects of prefeeding, intercomponentinterval food, and extinction on temporal discrimination and pacemaker rate. Behavioural Processes, 71, 297-306.

Wilkie, D. M., Symons, L. A., \& TeEs, R. C. (1988). Effects of inter- trial reinforcers on rats' timing behavior. Behavioural Processes, 17, 229-238.

WILKIE, D. M., \& Willson, R. J. (1990). Discriminal distance analysis supports the hypothesis that pigeons retrospectively encode event duration. Animal Learning \& Behavior, 18, 124-132.

Wixted, J. T., \& Gaitan, S. C. (2004). Stimulus salience and asymmetric forgetting in the pigeon. Animal Learning \& Behavior, 32, 173-182.

Zentall, T. R. (2006). Timing, memory for intervals, and memory for untimed stimuli: The role of instructional ambiguity. Behavioural Processes, 71, 88-97.

(Manuscript received August 9, 2006; accepted for publication October 19, 2006.) 